\title{
Industrial aluminum hazardous waste as a new raw material for zeolite synthesis
}

\author{
A. López-Delgado ${ }^{1}$, O. Rodríguez ${ }^{1}$, I. Padilla $^{1}$, R. Galindo $^{1} \&$ \\ S. López-Andrés ${ }^{2}$ \\ ${ }^{1}$ National Centre for Metallurgical Research, Spain \\ ${ }^{2}$ Faculty of Geology, Complutense University of Madrid, Spain
}

\begin{abstract}
In Spain, the secondary aluminum industry is a mature activity which produces $35 \%$ of the total aluminum production. This industry generates slag (black dross) with a valuable content of aluminum which is recovered by dry milling processes in the aluminum tertiary industry. The powdered fine solid is trapped in the sleeve filter suction system of the aluminum slag milling process. This solid is considered as a hazardous waste within the 100321 group in the European Waste Catalogue (EWC), because of the very fine grain size $\left(\mathrm{x}_{50}<50 \mu \mathrm{m}\right)$ and the presence of dangerous substances (metallic aluminum, AlN, heavy metals, etc.). The high aluminum nitride content, ranging between 2 and $24 \%$, is remarkable and it releases ammonia gas in the presence of environmental humidity. The aim of this work was to evaluate the complete recovery of the fine powders coming from the sleeve filters in the aluminum tertiary industry, as a zeolite. Two types of zeolite, analcime and $\mathrm{NaP}$ were obtained by hydrothermal processes from the aluminum waste and silica as raw materials. Different compositions of wastes and parameters such as time and temperature were assayed to study their effects on the obtained zeolites. The materials were characterized by X-ray diffraction and scanning electron microscopy.

Keywords: aluminum hazardous waste, recycling, zeolite, analcime, NaP.
\end{abstract}

\section{Introduction}

Aluminum recycling is a large chain formed by several industries, which recovers this metal from wastes produced. Particularly, the secondary industry smelts waste such as scrap, slag, dross and skimming but generates the black 
dross as an undesired by-product; a kind of aluminum slag with high content of molten salts. In Spain, this industry produces around $35 \%$ of the total aluminum production. According to basically economical aspects, the disposal into landfills is mostly the final destination of the black dross, as Tsakiridis et al. [1] described, but in many cases, dry milling processes are performed in order to recover the largest fraction of the remaining aluminum. These milling processes take place in other industries which are known as the aluminum tertiary industry.

Despite the benefits of milling, certain wastes are generated. Specifically, a fine powder is trapped into the sleeve filters of the suction systems. This solid is considered as a hazardous waste according to the European Waste Catalogue (EWC) within the 100321 group and currently, is sent to landfill instead of recovered. The main features of this powder are the extremely fine grain size $\left(\mathrm{x}_{50}<50 \mu \mathrm{m}\right)$ and the presence of dangerous substances such as aluminum nitride (AlN), metallic aluminum, heavy metals, etc. In this sense, the high content of aluminum nitride is highly remarkable (ranging between 2 and $24 \%$ in weight) because this substance often interacts with the environmental humidity or water releasing ammonia gas in considerable quantities [2, 3]. The recent attempts performed to recover this waste are based on the production of viable added-value materials in order to overcome the economical barrier which prevents the implementation of conventional recovery processes [4].

Zeolites are an extensively studied group of aluminosilicates which are formed by three dimensional networks of $\mathrm{AlO}_{4}$ and $\mathrm{SiO}_{4}$ tetrahedra linked together by shared oxygen atoms [5]. The high microporosity present in these materials ("molecular sieve"), the number of documented methods to obtain them and the possibility to introduce variations on their compositions as well as morphologies [6] allow a huge number of applications in interesting fields such as catalysis, ion exchange, chemical separation, membranes, etc. Inside this group of porous materials, $\mathrm{NaP}$ and analcime are recognized by their excellent properties as ion exchangers. Both zeolites are formed in the system $\mathrm{Na}_{2} \mathrm{O}-\mathrm{Al}_{2} \mathrm{O}_{3}-\mathrm{SiO}_{2}-\mathrm{H}_{2} \mathrm{O}$. Thus, the gismondine-type structure is present for the $\mathrm{NaP}[7,8]$ and analcime adopts different crystal symmetries as a function of the hydrothermal synthesis temperatures, being for instance orthorhombic at lowest temperatures and tetragonal or cubic depending on whether it rises [9].

Methods to obtain zeolites from unconventional sources and innovative techniques have been described in the literature. In particular, certain studies are based on the introduction of hazardous wastes as raw material in order to reduce their disposal into landfills. Examples such as the use of asbestos or fly ash from carbon combustion plants and municipal solid waste incinerators are remarkable $[10,11]$. However, as far as we know, the use of aluminum hazardous waste from the tertiary industry has not been described

Therefore, the aim of this work was to evaluate the complete recovery of the fine powders coming from the sleeve filters in the aluminum tertiary industry in order to obtain an added-value material as a zeolite. Thus, two types of zeolite, analcime and $\mathrm{NaP}$ were obtained by hydrothermal process from the aluminum waste and silica as raw materials. Different compositions and parameters such as 
time and temperature were assayed to study their effect on the resulting zeolites. The materials were characterized by X-ray diffraction and scanning electron microscopy.

\section{Experimental methods}

Eleven different bulk samples (W1 to W11) of waste coming from the sleeve filter suction system of the aluminum slag milling process were provided by two tertiary industries in Madrid and Zaragoza (Spain). Wastes, which consisted of very fine and grey colored powders (with a characteristic odor due to the chemical composition), were divided into representative samples by a Laborette 27, a rotary cone sample divider (Fig. 1). Samples were characterized as follows: Particle size distribution was carried out using a Sympatec Helos 12LA and an ultrasonic dispersion of waste in isopropyl alcohol. The chemical composition was analyzed by X-ray fluorescence (XRF), employing a wavelength dispersive X-ray fluorescence spectrometer (Bruker, S8 Tiger). Measurements were acquired using fusion disks prepared with lithium tetraborate and lithium metaborate at $60 \mathrm{k} \mathrm{V}$ and $170 \mathrm{~mA}$. The main crystalline phases present in wastes were determined by X-ray diffraction (XRD) using a Bruker D8 Advance Diffractometer with $\mathrm{CuK} \alpha$ radiation, from $5^{\circ}$ to $85^{\circ} 2 \theta$, at a scan rate of $0.02^{\circ} 2 \theta$, 5 s per step, $40 \mathrm{kV}$ and $30 \mathrm{~mA}$. The aluminum nitride content, one of the main components responsible for the waste hazardousness, along with metallic aluminum [2, 4], was analyzed by the Kjeldhal method using an automatic steam distilling unit UDK 130 A by Velp Scientifica, and subsequent titration with $1 \mathrm{M} \mathrm{HCl}$.

\subsection{Zeolite synthesis}

The synthesis of zeolite from the aluminum waste was carried out by a conventional hydrothermal method, employing a Teflon-lined autoclave (1 liter of capacity). Taking into account future large scale applications, a simple onestep zeolitification process without a previous alkalinization/activation step was employed. In this sense, the raw materials selected for supplying the required amount of $\mathrm{Al}_{2} \mathrm{O}_{3}$ in the zeolite synthesis were waste. By this way, minor amounts of $\mathrm{SiO}_{2}$ and $\mathrm{Na}^{+}$also were introduced. The $\mathrm{SiO}_{2}$ and $\mathrm{Na}^{+}$balance was established by adding commercial silica and the activating aqueous solution, $0.5 \mathrm{M} \mathrm{NaOH}$ (both reagents provided by Panreac, S.A) being the $\mathrm{Al}_{2} \mathrm{O}_{3} / \mathrm{SiO}_{2}$ ratio equal to $6 / 10$ in all tests. The reactions took place in the autoclave for 3,6 and $18 \mathrm{~h}$. Temperature conditions ranged between 100 and $200^{\circ} \mathrm{C}$ and the autogeneous pressure was maintained at 10-12bar. When reaction time finished, products were filtered on a GTTP Millipore filter of $0.22 \mu \mathrm{m}$ and washed thrice with distilled water. Finally, they were dried in air at $80^{\circ} \mathrm{C}$ for $24 \mathrm{~h}$. Solid products were characterized by XRD and scanning electron microscopy (SEM) using a Hitachi S4800 (15.0 kV, $14.8 \mathrm{~mm})$. 


\section{Results and discussion}

Table 1 presents the average chemical composition (main components) of the aluminum wastes with statistical values such as: mean value $(\overline{\mathbf{x}})$ standard deviation $(\sigma)$ minimum and maximum values $(\min , \max )$ and mode obtained from the eleven samples analyzed. Minority components such as copper, lead, chromium, manganese, sulfide, etc., are included all together in the column denoted by "others". As can be seen, a high deviation in the chemical composition appears, being product of the variable composition present in the raw slag. In other words, this fact is a direct consequence of the high heterogeneity present in all samples.

Table 1: Statistical values of waste chemical composition (main components) obtained by XRF (wt. \%) and contents of aluminum nitride (AIN) calculated by Kjeldhal method (wt. \%).

\begin{tabular}{|l|c|c|c|c|c|c|c|c|c|c|c|}
\hline Compound & $\mathrm{Al}_{2} \mathrm{O}_{3}$ & $\mathrm{MgO}$ & $\mathrm{Fe}_{2} \mathrm{O}_{3}$ & $\mathrm{TiO}_{2}$ & $\mathrm{SiO}_{2}$ & $\mathrm{CaO}$ & $\mathrm{K}_{2} \mathrm{O}$ & $\mathrm{Na}_{2} \mathrm{O}$ & $\mathrm{Cl}$ & Others & $\mathrm{AlN}$ \\
\hline$\overline{\mathrm{x}}$ & 75 & 4.0 & 1.4 & 2.8 & 6.3 & 2.8 & 0.7 & 2.2 & 1.2 & 3.3 & 13.3 \\
$\sigma$ & 8 & 1.2 & 1.0 & 2.3 & 4.7 & 1.1 & 0.7 & 2.7 & 1.2 & 1.3 & 5.4 \\
$\min$ & 53.38 & 1.32 & 0.35 & 0.40 & 2.56 & 1.64 & 0.23 & 0.00 & 0.01 & 0.97 & 1.40 \\
$\max$ & 80.96 & 6.76 & 3.44 & 8.30 & 17.79 & 5.97 & 2.98 & 10.46 & 3.49 & 5.69 & 23.60 \\
mode & 71 & 4.2 & 0.7 & 1.1 & 2.9 & 2.2 & 0.4 & 1.6 & 0.7 & --- & 13.7 \\
\hline
\end{tabular}

Concerning the granulometry, the wastes consisted of very fine size grain samples with $\mathrm{x}_{50}$ values smaller than $30 \mu \mathrm{m}$. The statistical values of particle size distribution determined for all samples are shown in Table 2. As a consequence of the intense process of milling performed to recovery these wastes, the high reduction in size generates powders with a high volatile component which is trapped in the sleeve filters and is also responsible for the waste hazardousness due to the high possibilities to cause severe health effects after prolonged exposure.

Table 2: $\quad$ Statistical values of particle size distribution of the aluminum solid wastes.

\begin{tabular}{|l|c|c|c|}
\hline data & $\mathrm{x}_{10}(\mu \mathrm{m})$ & $\mathrm{x}_{50}(\mu \mathrm{m})$ & $\mathrm{x}_{90}(\mu \mathrm{m})$ \\
\hline$\overline{\mathrm{x}}$ & 2.5 & 22.8 & 92.8 \\
$\sigma$ & 1.7 & 11.8 & 39.9 \\
$\min$ & 0.9 & 5.1 & 40.4 \\
$\max$ & 7.1 & 47.4 & 164.0 \\
mode & 2.4 & 27.7 & 81.2 \\
\hline
\end{tabular}


The particle size distribution, determined by laser diffraction, showed for the most of wastes a trimodal tendency. Several examples of the distribution density curves are collected in Figure 1 (samples W1, W2, W7, and W11). As it can be seen, the W11 sample showed a quite different curve with a multimodal profile. In this case was remarkable that peaks corresponding to small, medium and large sizes appeared within a small range of distribution density. Moreover, sample presented a maximum value of $164.0 \mu \mathrm{m}$ for $\mathrm{x}_{90}$ as can be appreciated in Table 2 . This fact is related to the highest metallic aluminum content present in this sample as below mentioned, which forms spherical particles that are more hardly crushed in the milling process in comparison with the other oxidized phases and salts.

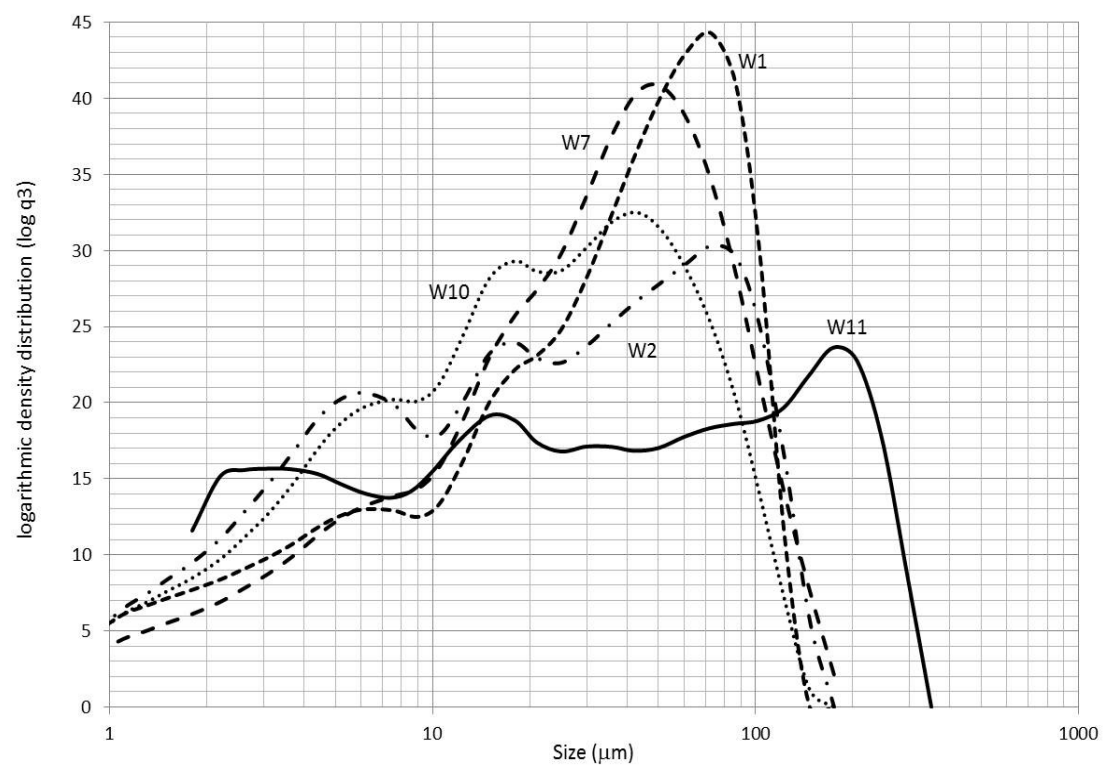

Figure 1: $\quad$ Particle size distribution curves of selected aluminum waste.

From a mineralogical point of view, wastes comprised a mixture of both amorphous and crystalline phases. The crystalline ones were mainly composed of not well defined peaks, with variable intensity, which were assigned to aluminum phases such as corundum, aluminum nitride, metallic aluminum and spinel, along with them, other phases as quartz, calcium carbonate and $\mathrm{Na} / \mathrm{K}$ chloride were detected. As an example for the XRD patterns, two different wastes are shown in Figure 2. It is noticeable the high intensity of the peaks corresponding to the metallic aluminum for sample W11, which are located at 38.4 and $44.6^{\circ}$ (20) (see Figure $2 b$ ).

For the experiments of zeolite synthesis performed at $100^{\circ} \mathrm{C}$, all formed products presented disordered amorphous phases which could not be identified by XRD. In the case of products obtained at $150^{\circ} \mathrm{C}$, peaks appeared with very low 

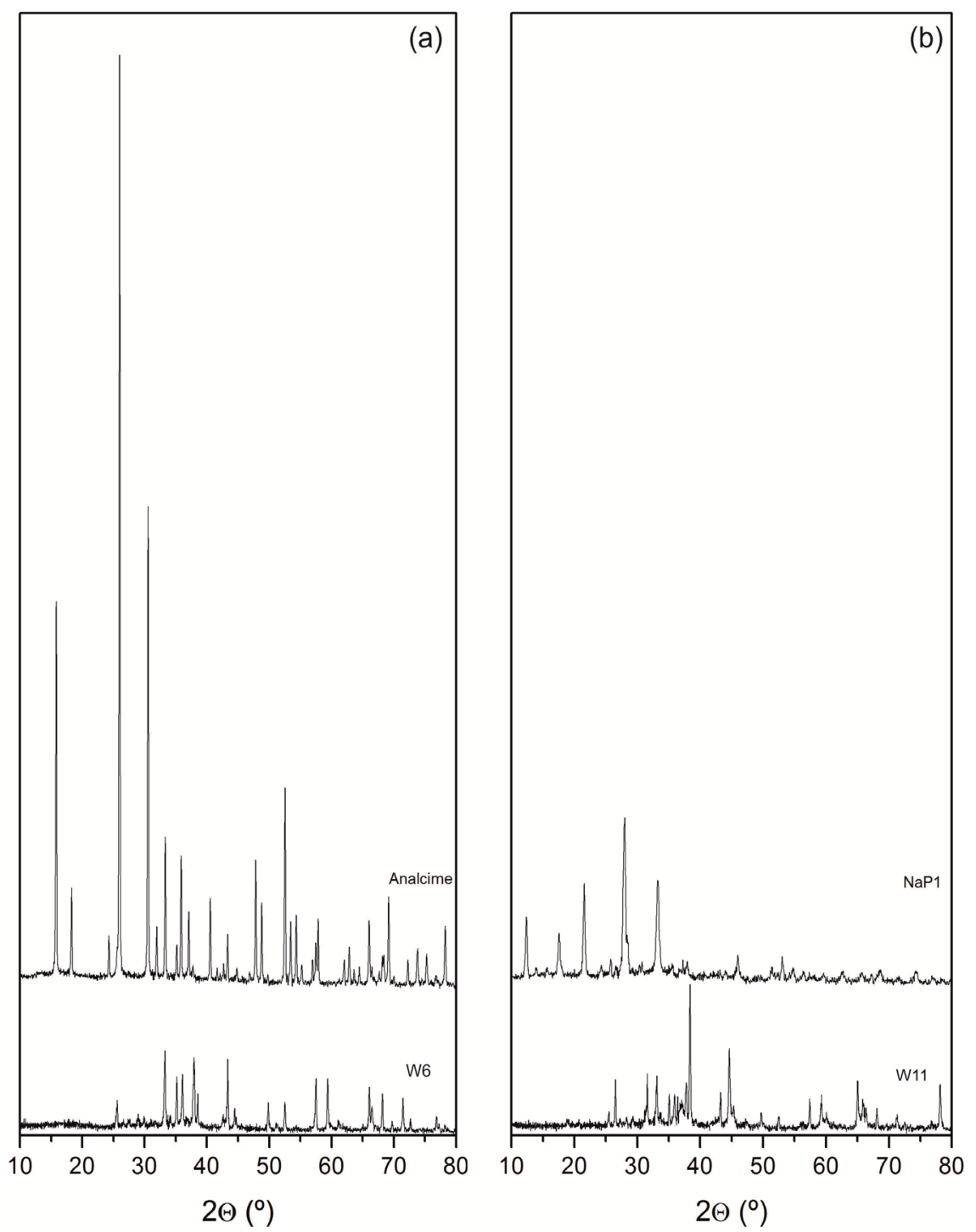

Figure 2: $\quad \mathrm{XRD}$ patterns of waste samples (W6 and W11) and the zeolites: (a) analcime [JCPDS 00-041-1478]; (b) NaP1 [JCPDS 01-0710962].

intensity, which were attributable to zeolitic materials. When essays were conducted at $200^{\circ} \mathrm{C}$, all the products exhibited XRD patterns corresponding to defined crystalline phases of zeolite. These last findings were observed for essays conducted to a reaction time of $6 \mathrm{~h}$. For the shortest time $(3 \mathrm{~h}), \mathrm{XRD}$ patterns consisted of amorphous phases, and for the longest time (18 h), differences were not found respect to the essays conducted to $6 \mathrm{~h}$. Figure 2 
shows the diffraction patterns of the zeolites corresponding to analcime and $\mathrm{NaP}$ which were prepared at same experimental conditions: $200^{\circ} \mathrm{C}, 6 \mathrm{~h}$ and $0.5 \mathrm{M}$ $\mathrm{NaOH}$ solution, using two different wastes (W6 and W11). The XRD pattern of analcime, $\mathrm{Na}\left(\mathrm{Si}_{2} \mathrm{AlO}_{6}\right) \cdot \mathrm{H}_{2} \mathrm{O}$, fitted well with the JCPDS reference (00-041-1478) and the $\mathrm{NaP} 1$ zeolite pattern, $3 \mathrm{Na}_{2} \mathrm{O} \cdot 3 \mathrm{Al}_{2} \mathrm{O}_{3} \cdot 10 \mathrm{SiO}_{2} \cdot 15 \mathrm{H}_{2} \mathrm{O}$, with JCPDS 01-071-0962. In this figure, the axe for intensities was the same in all samples, in order to a better comparison of the different degree of crystallinity between wastes and zeolites.

As it can be seen, the pattern of analcime corresponds to a well crystallized sample with very high intensity and well defined peaks. On the contrary, the diffraction peaks corresponding to $\mathrm{NaP} 1$ present a lower intensity which indicates smaller crystallite size and lower crystallinity. All waste samples were tested in those experimental conditions. Analcime was obtained in all cases except when W11 was used. This finding can be associated with the abovementioned characteristics present in this sample such as the more uniform distribution of grain size and also the highest content of metallic aluminum which favors the higher cumulative distribution at $90 \%\left(\mathrm{x}_{90}\right)$. The hydrolysis rate for the different aluminum compounds (alumina, aluminum nitride, metallic aluminium, etc.) would be responsible on the different results. Thus, the kinetics of the hydrolysis suffers variations when higher amount of metallic aluminum is present, being much faster in comparison to the rest of aluminum compounds [12]. The faster kinetics favors the formation of $\mathrm{NaP}$ zeolite.

The macroscopic appearance of a waste sample used as synthesis precursor, the as-obtained wet zeolite after filtration and the dried zeolite are shown in Figure 3. The visual aspect for the wet product presented highly porous space between particles after filtering; this situation was coherent with the subsequent spongy and non-compacted texture in the case of the dry zeolite.

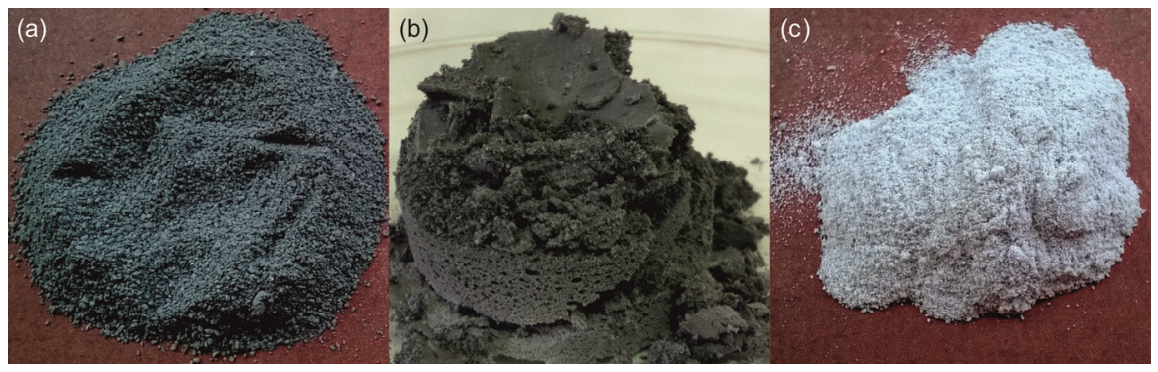

Figure 3: (a) Macroscopic appearance of an initial waste sample; (b) the as-obtained wet zeolite after filtering; (c) the corresponding dried zeolite.

The aluminum content in the remaining solution after filtration was analyzed by atomic absorption spectroscopy (AAS, (EspectrAA 220 FS, Varian Techtron, Australia Ply Ltd), being equal to $37 \mathrm{mg} / \mathrm{l}$. This result indicated that $99.99 \%$ of the introduced aluminum from waste was transformed into zeolite. 
The morphology of the analcime which exhibited trapezohedral crystals is shown in Figure 4. As can be seen at highest magnification, this zeolite-type presented well-formed pentagonal icositetrahedrons composed of 24 trapezohedral faces with clear crystal edges. Related to NaP1 zeolite (see Figure 5), SEM images showed agglomerates formed by tiny cubic crystallites. This fact was in agreement with the XRD results. In this sense, different morphologies for $\mathrm{NaP1}$ have been obtained depending on the synthesis conditions which are documented in the literature $[6,8]$.

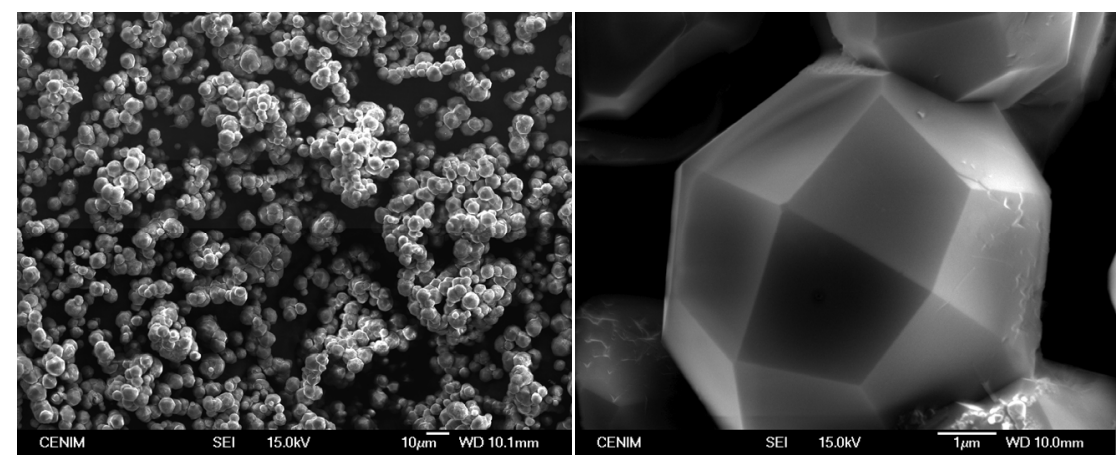

Figure 4: SEM images of analcime magnified at: (left) x500; (right) x13000.

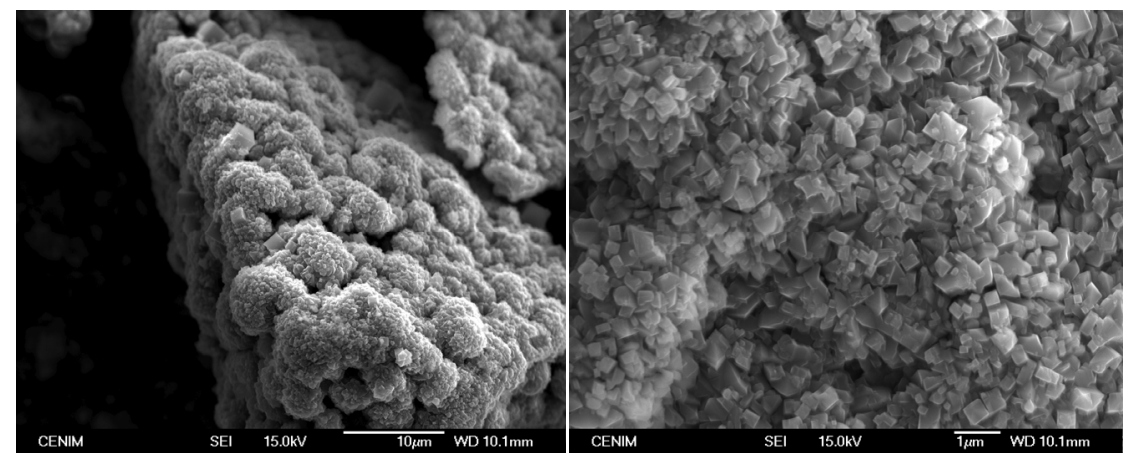

Figure 5: $\quad$ SEM images of NaP1 magnified at: (left) x2200; (right) x10000.

\section{Conclusions}

Samples of hazardous wastes from the aluminum tertiary industry were used as aluminum raw material for the preparation of zeolites. The synthesis method was based on a conventional alkaline hydrothermal process. A simple one-step zeolitification process was employed in order to decrease the process cost. 
Two zeolite analcime and $\mathrm{NaP}$ were obtained depending on the temperature, time and waste composition. The higher granulometry and presence of highest metallic aluminum favored the $\mathrm{NaP}$ zeolite in comparison to analcime. This last exhibited a higher crystallinity than $\mathrm{NaP}$.

The process allows the complete recovery of the industrial aluminium hazardous waste as a valuable material. Future research is going to be focused towards the application of the zeolites obtained.

\section{Acknowledgements}

Authors thank the MINECO (Spain) for financing the project CTM2012-34449 and the companies Recuperaciones y Reciclajes Roman S.L (Fuenlabrada, Madrid, Spain) and Metalquex, S.L. (Zaragoza, Spain) for supplying the wastes.

\section{References}

[1] Tsakiridis, P.E., Oustadakis, P. \& Agatzini-Leonardou, S., Aluminum recovery during black dross hydrothermal treatment, Journal of Environment Chemical Engineering, 1, pp. 23-32, 2013.

[2] Krnel, K., Drazic, G., \& Kosmac, T., Degradation of AlN powder in aqueous environments, Journal of Materials Research, 19(4), pp. 1157-1163, 2004.

[3] Galindo, R., Padilla, I., Rodríguez, O., López-Delgado, A, Shinzato, M.C., Braz, I.G., Carvalho, F.M.S. \& López-Andrés, S., Current state of the hazardous waste recycling in the aluminium metallurgy in Spain and Brazil, Waste Materials and Research, in press, 2014.

[4] López-Delgado, A. \& Tayibi, H., Can hazardous waste become a raw material? The case study of an aluminium residue: a review, Waste Management and Research, 30(5), pp. 474-484, 2012.

[5] Sherman, J.D., Synthetic zeolites and other microporous oxide molecular sieves, Proceedings of the National Academy of Science of the United States of America, 96, pp. 3471-3478, 1999.

[6] Huo, Z., Xu, X., Lü, Z., Song, J., He, M., Li, Z., Wang, Q \& Yan, L., Synthesis of zeolite NaP with controllable morphologies, Microporous and Mesoporous Materials, 158, pp. 137-140, 2012.

[7] Hansen, S.H., Håkansson, U., Landa-Canovas, A.R. \& Fälth, L., On the crystal chemistry of NaP zeolites, Zeolites, 13, pp. 276-280, 1993.

[8] Albert, B.R., Cheetham, A.K., Stuart, J.A \& Adams, C.J., Investigations on $P$ zeolites: synthesis, characterisation, and structure of highly crystalline low-silica NaP, Microporous and Mesoporous Materials, 21, pp. 133-142, 1998.

[9] Ghobarkar, H. \& Schäf, O., Effect of temperature on hydrothermal synthesis of analcime and viséite, Materials Science and Engineering B, 60, pp. 163-167, 1999. 
[10] Querol, X., Plana, F., Alastuey, A. \& López-Soler, A., Synthesis of Nazeolites from fly ash, Fuel, 76(8), pp. 793-799, 1997.

[11] Yang, G.C.C \& Yang, T-Y., Synthesis of zeolites from municipal incinerator fly ash, Journal of Hazardous Materials, 62, pp. 75-89, 1998.

[12] López-Delgado, A., Tayibi, H. \& López, F.A., Treatments of aluminium dust: a hazardous residue from secondary aluminium industry (Chapter 1). Focus on Hazardous Materials Research, ed. L.G. Mason, Nova Science Publishers, Inc: New York, pp. 1-52, 2007. 